\title{
Adsorção de fósforo em solos de regiões tropicais
}

\author{
Ana Paula Carrara VINHA ${ }^{*}$, Bruna Helena CARRARA ${ }^{1}$, Emanuella Farias Santos SOUZA ${ }^{1}$, \\ Jussane Antunes Fogaça dos SANTOS ${ }^{1}$, Sayonara Andrade Couto Moreno ARANTES 1
}

${ }^{1}$ Instituto de Ciências Agrárias e Ambientais, Universidade Federal de Mato Grosso, Sinop, MT, Brasil. (Orcid: 0000-0002-8462-8983; 0000-0002-5866-1819; 0000-0001-9109-2163; 0000-0001-5089-2260; 0000-0002-1559-1021)

*E-mail: anapcarrara@gmail.com

Recebido em 13/08/2020; Aceito em 22/12/2020; Publicado em 10/02/2021.

\begin{abstract}
RESUMO: O fósforo (P) é o nutriente mais limitante ao cultivo nos solos tropicais, que, geralmente, apresentam alta capacidade de adsorção de P. Alguns atributos químicos e físicos do solo como o teor de argila, pH, CTC efetiva e matéria orgânica influenciam na dinâmica de adsorção. O objetivo deste estudo foi avaliar o processo de adsorção do fósforo em um Latossolo Vermelho Amarelo (LVA) e um Neossolo Quartzarênico (RQ) da região de transição Cerrado-Amazônia, e avaliar os atributos dos solos que influenciam na dinâmica de adsorção do P. Os ensaios de sorção foram realizados pelo método "Batch" ou em batelada e à partir dos dados obtidos foram construídas curvas obtendo a equação linearizada de Langmuir, com as quais foram determinados os valores de capacidade máxima de adsorção de P (CMAP), a constante relacionada com a energia de ligação $\left(\mathrm{K}_{\mathrm{L}}\right)$ e o fator capacidade de $\mathrm{P}$ máximo ( $\mathrm{FCP}_{\text {máx }}$ ). Houve diferença na $\mathrm{CMAP}, \mathrm{K}_{\mathrm{L}}$ e $\mathrm{FCP}_{\text {máx }}$ dos solos estudados, sendo que os maiores valores foram encontrados no LVA, devido principalmente ao maior teor de argila em relação ao RQ. A matéria orgânica foi um dos poucos atributos que tiveram correlação negativa com os atributos de adsorção, ressaltando sua importância no manejo para aumentar a eficiência da adubação fosfatada.
\end{abstract}

Palavras-chave: Latossolo Vermelho Amarelo; Neossolo Quartzarênico; Isotermas de adsorção.

\section{Phosphorus adsorption in soils of tropical regions}

\begin{abstract}
Phosphorus $(\mathrm{P})$ is the most limiting nutrient for cultivation in tropical soils, as they generally have high $\mathrm{P}$ adsorption capacity. Some chemical and physical attributes of soil such as clay content, $\mathrm{pH}$, effective cation exchange capacity (ECEC) and organic matter influence the adsorption dynamics. The objective of this study was to evaluate the behaviour of the phosphorus adsorption process in a Red-Yellow Latosol and a Quartzarenic Neosol from the Cerrado-Amazon transition region, and to evaluate the soil attributes that influence the adsorption dynamics of the soil of the phosphorus. Sorption tests were performed using the "Bach" method, from the Langmuir linearized equation, the maximum P adsorption capacity (MPAC), the constant with the binding energy $\left(\mathrm{K}_{\mathrm{B}}\right)$ and the maximum capacity factor (PCFmax) were selected. There was a difference in MPAC, $K_{B}$ and PCFmax of the studied soils, and the highest values were found in the Red-Yellow Oxisol, mainly due to the higher clay content in relation to theTypic Quartzipisamment. Organic matter was one of the few attributes that had a negative correlation with the adsorption attributes, highlighting (emphasizing) it's importance in (soil) management to increase the efficiency of phosphate fertilization.

Keywords: Red-Yellow Latosol; Quartzarenic Neosol; Adsorption isotherms.
\end{abstract}

\section{INTRODUÇÃO}

De acordo com o processo de intemperismo, um solo pode alterar sua função de fonte ou dreno de fósforo $(\mathrm{P})$ para atender as necessidades das plantas ou competir com elas, fixando o $\mathrm{P}$ adicionado ao solo em sítios ativos. O aumento da capacidade de adsorção de ânions ocorre por meio de sítios ativos positivos, formados principalmente por hidróxidos de ferro e alumínio (FARIAS et al., 2009).

Os processos de adsorção e dessorção de $\mathrm{P}$ do solo consideram dois compartimentos interligados, a solução do solo e a fase sólida, essa última armazena o nutriente que abastece a primeira (DE OLIVEIRA et al., 2014).
Segundo Parfitt (1978), o processo de adsorção de P ocorre na forma binucleada em três estádios temporais, conforme tempo de reação. No estádio 1 , predominam a neutralização das cargas positivas (grupos $\mathrm{R}_{-} \mathrm{OH}_{2}{ }^{+}$), neste estádio a reação dá-se de forma rápida e fortemente em sítios muito reativos. No estádio 2 , o P é adsorvido em sítios menos reativos. Já no estádio 3, a longo prazo, há interações de caráter eletrostático entre o $\mathrm{PO}_{4}$ e a superfície dos coloides, migrando para lóbulos internos dos cristais, podendo ser considerado como precipitação.

Os estádios estão relacionados com a afinidade entre o P e os sítios de adsorção em cada fase e, consequentemente, 
com distintas energias de ligação. Como pressupostos do mecanismo de adsorção, os sítios de adsorção do P devem ser isolados e independentes quanto à energia de retenção e que as moléculas adsorvidas não devem interagir umas com as outras (DE OLIVEIRA et al., 2014).

Vários modelos matemáticos são utilizados para descrever as proporções de $\mathrm{P}$ adsorvido à fase sólida em razão da adição de quantidades crescentes de $\mathrm{P}$ na solução. $\mathrm{O}$ uso da isoterma de Langmuir tem sido comum na determinação desses fatores, uma vez que contém um parâmetro quantitativo relacionado a capacidade máxima de adsorção de fósforo (CMAP) e um parâmetro qualitativo que expressa a energia de ligação entre ânions e locais de adsorção, a constante "k" (FARIAS et al., 2009; MATOS et al., 2017). Podendo ser relacionada as propriedades dos solos para obtenção de informações sobre a natureza das reações entre o solo e o P adicionado.

A intensidade da adsorção de P no solo é relacionada com a quantidade em que ele está presente ou é adicionado, promovendo um equilíbrio entre os dois fatores. Em solos argilosos é característico uma maior adsorção de $\mathrm{P}$, principalmente os mais intemperizados, onde a relação quantidade e intensidade é maior, quando comparado a um

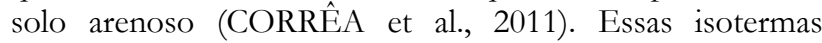
revelam que a adsorção de $\mathrm{P}$ aumenta com o aumento da concentração do elemento em solução de equilíbrio.

Em solos altamente intemperizados a eficiência da adubação fosfatada é, geralmente, baixa, pois grande parte do $P$ adicionado é imobilizada no solo, em virtude de reações de precipitação, adsorção e fixação em coloides minerais (PELUCO et al., 2015)

Tabela 1. Atributos químicos e físicos do Latossolo Vermelho Amarelo (LVA) e do Neossolo Quartzarênico (RQ). Table 1. Chemical and physical attributes of Red-Yellow Latosol and Quartzarenic Neosol.

\begin{tabular}{|c|c|c|c|c|c|c|c|c|c|c|c|c|}
\hline \multicolumn{13}{|c|}{ Análises químicas } \\
\hline \multirow{2}{*}{ Solo } & $\mathrm{pH} \mathrm{H}_{2} \mathrm{O}$ & $\mathrm{pHCaCl}_{2}$ & $\mathrm{P}$ & $\mathrm{K}^{+}$ & $\mathrm{Ca}^{+2}$ & $\mathrm{Mg}^{+2}$ & $\mathrm{Al}^{+3}$ & $\mathrm{H}^{+}$ & CTCefet. & CTCpot. & $\mathrm{V}$ & MO \\
\hline & \multicolumn{4}{|c|}{-------------- $\left(\mathrm{mg} \mathrm{dm}^{-3}\right)$-------------- } & \multicolumn{6}{|c|}{ 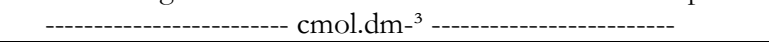 } & $\%$ & g.dm ${ }^{3}$ \\
\hline LVA & 4,6 & 4,0 & 0,94 & 13 & 0,57 & 0,21 & 1,00 & 6,36 & 1,81 & 8,17 & 9,92 & 25,03 \\
\hline RQ & 4,4 & 3,8 & 1,38 & 11 & 0,13 & 0,03 & 1,35 & 5,96 & 1,54 & 7,50 & 2,56 & 25,84 \\
\hline \multicolumn{13}{|c|}{ Análises físicas } \\
\hline \multirow{2}{*}{ Solo } & \multirow{2}{*}{\multicolumn{4}{|c|}{ Areia }} & \multicolumn{4}{|c|}{ Silte } & \multicolumn{4}{|c|}{ Argila } \\
\hline & & & & & & $-\mathrm{mg} \mathrm{c}$ & $a^{3}-\cdots$ & & & ----- & & \\
\hline $\mathrm{LVA}$ & \multicolumn{4}{|c|}{330} & \multicolumn{4}{|c|}{188} & \multicolumn{4}{|c|}{482} \\
\hline RQ & \multicolumn{4}{|c|}{788} & \multicolumn{4}{|c|}{63} & \multicolumn{4}{|c|}{149} \\
\hline
\end{tabular}

Os solos coletados foram secos ao ar e peneirados em malha de $2 \mathrm{~mm}$. Para as análises de adsorção do fósforo, utilizou-se 2 gramas destas amostras e empregou-se o método de "Batch" (agitação durante 24 hrs à $120 \mathrm{rpm}$ ), onde para cada grama de solo utilizou-se $30 \mathrm{ml}$ de soluções de $\mathrm{P}$ nas seguintes concentrações: $0 ; 10 ; 20 ; 40 ; 80$ e $160 \mathrm{mg}$, preparadas em $\mathrm{CaCl}_{2} 0,01 \mathrm{M}$ e determinada a concentração em equilíbrio (Ce) em cada solução pura.

Após agitação, as soluções foram centrifugadas e os sobrenadantes foram utilizados para determinação do $\mathrm{P}$ por colorimetria, seguindo a metodologia de $\mathrm{P}_{\text {rem }}$ da Embrapa (2017). O P adsorvido (ADP) foi calculado pela diferença entre as quantidades de $\mathrm{P}$ adicionadas e as quantidades lidas na solução de equilíbrio do sobrenadante.

Com base nos resultados obtidos, construiu-se as isotermas de adsorção de $\mathrm{P}$, as quais representam graficamente a quantidade de $\mathrm{P}$ adsorvido na ordenada e sua Ce na abscissa. Utilizou-se a seguinte equação de Langmuir:
Dessa forma o objetivo deste estudo foi avaliar a adsorção do fósforo em um Latossolo Vermelho Amarelo e em um Neossolo Quartzarênico, presentes na região de transição Cerrado-Amazônia, e avaliar os atributos dos solos que influenciam na dinâmica de adsorção do P.

\section{MATERIAL E MÉTODOS}

O experimento foi conduzido no Laboratório de Solos da Universidade Federal de Mato Grosso (UFMT), em SinopMT. Os solos utilizados foram coletados em áreas de floresta nativa sendo um Latossolo Vermelho Amarelo (LVA), sob as

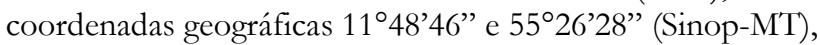
altitude 379, e o outro um Neossolo Quartzarênico (RQ) sob

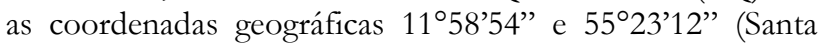
Carmem-MT), altitude 340.

O clima é considerado Aw (tropical com inverno seco), segundo a classificação de Koppen-Geiger, com duas estações definidas: seca de maio a setembro e chuvosa de outubro a abril. O bioma em que se encontra ambos os solos, é o de transição Cerrado/Amazônia e a pluviosidade média anual é de $1818 \mathrm{~mm}$. Nesta região as classes de solo mais representativas são Latossolo Vermelho Amarelo (LVA), Latossolos Vermelhos (LV), Neossolo Quartzarênico (RQ) e em menor proporção, Gleissolos $(\mathrm{G})$, oriundos de rochas sedimentares (RADAMBRASIL, 1982).

Foram coletadas 20 amostras simples para a composição de uma amostra composta de cada solo, coletados na camada superficial de 0 a $20 \mathrm{~cm}$ de profundidade. As análises químicas e físicas dos solos foram realizadas segundo Embrapa (2017), e estão contidas na Tabela 1.

$$
\begin{aligned}
& \frac{\mathrm{x}}{\mathrm{m}}=\frac{\mathrm{CMAP \cdot Ce \cdot \textrm {Kl }}}{1+\mathrm{Kl} \cdot \mathrm{Ce}} \\
& \frac{\mathrm{x}}{\mathrm{m}}=\frac{\mathrm{CMAP} \cdot \mathrm{Ce} \cdot \mathrm{Kl}}{1+\mathrm{K} \cdot \mathrm{Ce}}
\end{aligned}
$$

em que: $C M A P=$ capacidade máxima de adsorção de $\mathrm{P}, \mathrm{em}$ mmol por quilo de solo; $K l=$ constante relacionada com a energia de ligação (Constante de Lamgmuir) que dá a capacidade de adsorção teórica na monocamada $\left(\mathrm{L} \mathrm{Kg}^{-1}\right) ; C e=$ concentração de $\mathrm{P}$ na solução de equilíbrio $\left(\mathrm{mmol} \mathrm{P} \mathrm{L}^{-1}\right) ; x / m=$ quantidade de $\mathrm{P}$ adsorvida por $\mathrm{Kg}$ de solo $\left(\mathrm{mg} \mathrm{Kg}^{-1}\right)$. Os teores iniciais de $\mathrm{P}$ não foram adicionados aos valores de CMAP nos modelos matemáticos.

\section{RESULTADOS}

\subsection{Atributos químicos e físicos}

Pode-se observar na Tabela 1, que os valores de $\mathrm{pH}$ são baixos, característicos de solos ácidos e de elevado grau de intemperismo, típicos de ambientes tropicais. Isso sugere 
elevada acidez do solo e baixa disponibilidade de cátions básicos (MELO et al., 2010) além de gerar cargas positivas em minerais de argila de carga variável do solo, pois pode ocorrer a protonação das superfícies expostas destes minerais, principalmente dos óxidos. Os valores de $\Delta \mathrm{pH}$ são negativos $\left(\mathrm{pHCaCl}_{2}-\mathrm{pHH}_{2} \mathrm{O}\right)$, mostrando a predominância de cargas negativas.

A porcentagem de saturação por bases (V) foi baixa para ambos os solos ficando abaixo de $10 \%$ para os dois. Os teores de alumínio $\left(\mathrm{Al}^{3+}\right)$ e hidrogênio $\left(\mathrm{H}^{+}\right)$foram elevados, contribuindo para a baixa V. Portanto estes solos, em condições naturais, apresentam baixa capacidade em reter cátions $\left(\mathrm{Ca}^{2+}, \mathrm{Mg}^{2+}, \mathrm{K}^{+}\right)$e acidez excessiva. $\mathrm{O}$ teor de matéria orgânica (MO) foi de aproximadamente $25 \mathrm{~g} \quad \mathrm{dm}^{-3}$ comumente encontrados em solos tropicais de mata.

A classe textural (Tabela 1) foi arenosa para o RQ e argilosa para o LVA. Apesar dos pontos coletados serem próximos, ambos os solos estão situados numa bacia sedimentar, oriundos de rochas com proporções de minerais muito heterogênea originando solos com teores de argila distintos, muito característico da região. O conteúdo de argila influencia na capacidade de adsorção de $\mathrm{P}$, pois há maior quantidade de óxidos nesta fração, principalmente nos Latossolos.

\subsection{Isotermas de Langmuir}

A equação linearizada de Langmuir (Figura 1) apresentou alto coeficiente de correlação (Tabela 2), indicado que o modelo de Langmuir de adsorção foi significativamente capaz de estimar as constantes de adsorção de $\mathrm{P}$, em ambos os solos estudados.

Tabela 2. Equações da isoterma de Langmuir.

Table 2. Langmuir isotherm equations.

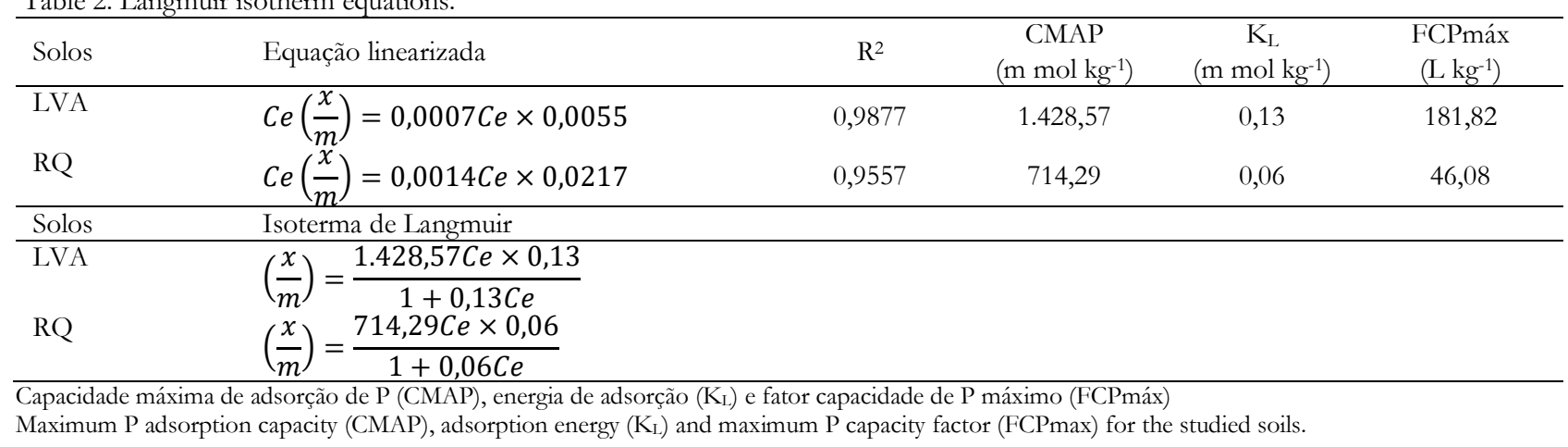

Na Figura 2, as curvas de adsorção obtidas pela equação de Langmuir ficaram muito próximas as médias das observações realizadas em laboratório. Nota-se no início da curva, uma inclinação acentuada, devido a atração eletrostática entre os $\mathrm{OH}^{-}$do íon fosfato e os $\mathrm{OH}_{2}{ }^{+}$das superfícies do solo (MELO; ALEONI, 2009), logo a curva tende a estabilizar-se num valor aproximadamente constante, concentração na qual todos os sítios de ligação ao fosfato estariam ocupados.

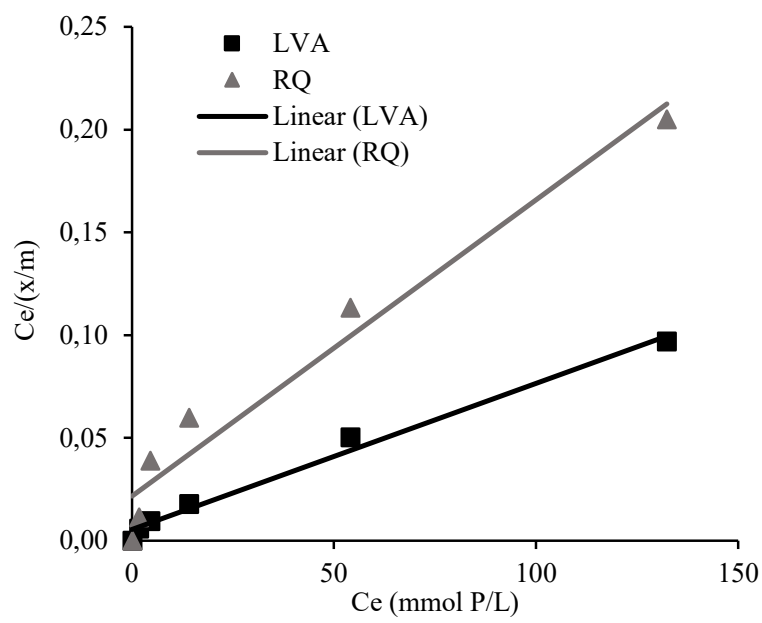

Figura 1. Isotermas de adsorção de Langmuir na forma linear. Ce: concentração de $\mathrm{P}$ na solução de equilíbrio; $\mathrm{Ce} /(\mathrm{x} / \mathrm{m})$ : relação entre Ce e $\mathrm{P}$ adsorvido no solo.

Figure 1. Langmuir adsorption isotherms in linear form. Ce: concentration of $\mathrm{P}$ in the equilibrium solution; $\mathrm{Ce} /(\mathrm{x} / \mathrm{m})$ : ratio between $\mathrm{Ce}$ and $\mathrm{P}$ adsorbed on soil.
Portanto, este modelo mostrou-se satisfatório e eficiente no estudo do fenômeno de adsorção de $\mathrm{P}$ ao solo, e capaz de estimar CMAP, FCP máx e $K_{L}$.

\subsection{Parâmetros de adsorção do $\mathbf{P}$}

Pelo modelo da isoterma de Langmuir foram calculados os parâmetros de adsorção, CMAP, $\mathrm{K}_{\mathrm{L}}$ e FCP máx (Tabela 2). O maior valor de CMAP foi verificado no LVA, 1.428,57 mmolP $\mathrm{Kg}^{-1}$ de solo, evidenciando a alta capacidade de sorção de P pelos Latossolos, enquanto que para o RQ foi de 714,29 mmolP $\mathrm{Kg}^{-1}$ de solo, essa grande variação está relacionada com o conteúdo de argila maior no LVA.

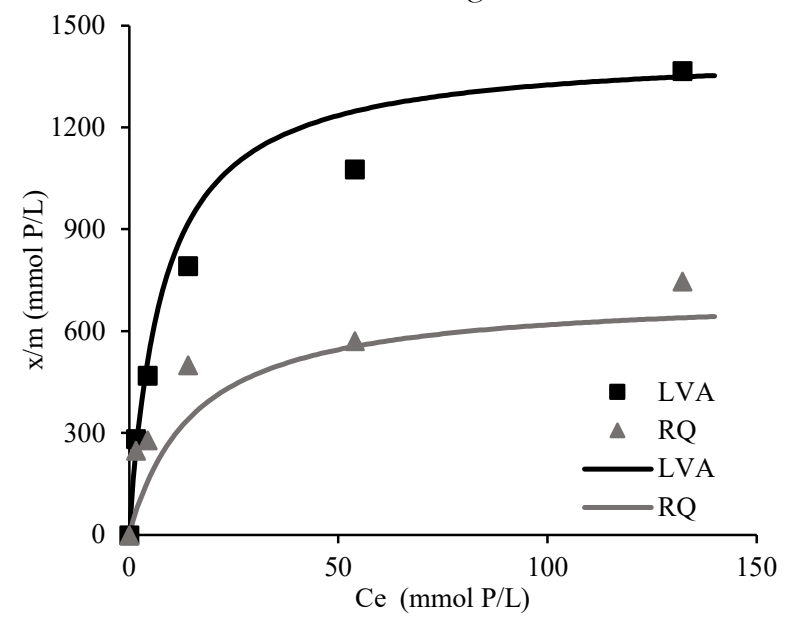

Figura 2. Isotermas de adsorção de fósforo. Ce: concentração de $\mathrm{P}$ na solução de equilíbrio; $\mathrm{x} / \mathrm{m}$ : P adsorvido no solo.

Figure 2. Phosphorus adsorption isotherms. Ce: concentration of $\mathrm{P}$ in the equilibrium solution; $\mathrm{x} / \mathrm{m}$ : P adsorbed on the soil. 


\subsection{Correlação entre os atributos do solo e adsorção}

Os parâmetros de adsorção foram correlacionados linearmente com alguns atributos do solo. Como foram avaliados apenas dois solos o coeficiente de correlação foi máximo para todos os atributos avaliados (Tabela 3). CMAP, $\mathrm{K}_{\mathrm{L}}$ e FCP $\mathrm{F}_{\text {máx }}$ correlacionaram-se positivamente com o teor de argila e o $\mathrm{pH}$, e negativamente com o conteúdo de $\mathrm{Al}^{+3}$ e MO.

Tabela 3. Correlação entre os parâmetros de adsorção e alguns atributos do solo.

Table 3. Correlation among the adsorption parameters and some soil attributes.

\begin{tabular}{lcccc}
\hline Parâmetro & Argila & $\mathrm{pHH}_{2} \mathrm{O}$ & $\mathrm{Al}^{+3}$ & $\mathrm{MO}$ \\
\hline CMAP (mmol) & 1 & 1 & -1 & -1 \\
$\mathrm{KL}$ & 1 & 1 & -1 & -1 \\
FCPmáx (L/kg) & 1 & 1 & -1 & -1 \\
\hline
\end{tabular}

1 - Correlação positiva; -1 - Correlação negativa.

1 - Positive correlation; - 1 - Negative correlation

\section{DISCUSSÃO}

O teor de MO encontrado é considerado adequado para solos tropicais (20-30 g. $\mathrm{dm}^{3}$ ), visto a taxa de mineralização acentuada influenciadas pela temperatura e umidade elevadas (CANTANRRUT, 2007b). A MO é a maior responsável pela fertilidade de solos tropicais, pela sua capacidade de quelar elementos tóxicos, fornece nutrientes através da mineralização e gerar cargas negativas (ROSS, 1993).

O conteúdo de argila influencia na capacidade de adsorção de fosforo, pois há alto conteúdo de óxidos nesta fração, principalmente nos latossolos (MOTTA et al., 2002).

O maior teor de óxidos nos latossolos juntamente com o maior teor de argila, é a provável razão pela qual a quantidade de fósforo (P) extraído foi menor no LVA que no RQ (BRADY; WEIL, 2013).

Neste estudo a isoterma de Langmuir se ajustou satisfatoriamente ao modelo de adsorção de P corroborando com resultados semelhantes encontrados por Guedes et al. (2015); Falcão; Silva (2004) e Matos et al. (2017), trabalharam com solos da região amazônica (Latossolo Amarelo, Latossolo Vermelho Amarelo, Argissolo Amarelo, Gleissolo Háplico, Nitossolo Vermelho, plintossolo Háplico e Planossolo Háplico), e obtiveram coeficientes de determinação mais elevados através da equação de Langmuir.

$\mathrm{A}$ adsorção do $\mathrm{P}$ no solo seguiu as pressuposições do modelo empírico de Langmuir, que são: os íons fosfato são adsorvidos em camada única na superfície dos coloides, e ocorre a adsorção máxima assim que a superfície é preenchida, sendo todos os sítios de adsorção idênticos de superfície homogênea e o fosfato adsorvido não interage com outras moléculas em solução, e o equilíbrio é atingido (OLIVEIRA et al., 2014).

Mesmo não sendo realizada a análise mineralógica dos solos, é fato que os latossolos tem predominância de óxidos em seus argilominerais, o que favorece a adsorção de P (BRADY; WEIL, 2013). Em estudo realizado por Wang et al. (2009), foi observado que o teor de óxidos metálicos (Fe e $\mathrm{Al}$ ) foi um fator principal que determinou a retenção de fósforo devido à alta superfície específica dos hidróxidos. Os autores avaliaram a cinética de adsorção do P e evidenciaram que o aumento da fração de argila com a maior área específica acelerou a adsorção.

Segundo RHEINHEIMER et al. (2003) em seu trabalho com Latossolo Vermelho Distroférrico típico, Latossolo Vermelho Distrófico típico e Argissolo Vermelho Distrófico típico cultivados sob sistema de plantio convencional e de plantio direto, o ajuste de isotermas sem considerar a quantidade prévia de fósforo sorvida pelo solo leva a interpretações errôneas da CMAP, devido às sucessivas aplicações de fertilizantes fosfatados. Nos sistemas cultivados, o fosfato adicionado e a elevação do $\mathrm{pH}$ via corretivos tende a saturar os sítios de maior sorção de $\mathrm{P}$, aumentando as cargas negativas semipermanentes $e$ diminuindo o potencial elétrico das partículas coloidais, quando o P migra para o interior dos óxidos (PARFFIT 1989). Porém utilizamos solos sob vegetação nativa, que nunca foram cultivados, os teores de P são "muito baixo" (SOUSA; LOBATO, 2004) portanto este parâmetro não foi levado em consideração no modelo matemático da CMAP.

Fang et al. (2017), estudaram o papel da morfologia de partículas na adsorção de $\mathrm{P}$ em que o mecanismo de adsorção é atribuído principalmente à carga superficial não uniforme e à heterogeneidade na superfície das partículas. Conforme aumenta a concentração de locais ativos de superfície aumenta a capacidade de adsorção.

Os valores de $K_{L}$ foram baixos (LVA: 0,13; e RQ: 0,06), o que evidencia que a adsorção de $\mathrm{P}$ está mais relacionada com a CMAP do que com a "K $\mathrm{K}_{\mathrm{L}}$ ", por esta razão que CMAP, isoladamente, tem sido utilizada com sucesso como padrão de recomendação da adubação fosfatada (FALCÃO; SILVA, 2004). Valores maiores de " $K_{\mathrm{L}}$ " são esperados em materiais mais argilosos, como constatado por Oliveira et al. (2014), em seu trabalho com um Latossolo Bruno eles alteraram a textura adicionando diferentes quantidades de areia a este mesmo solo, e obtiveram menor valor de " $\mathrm{K}_{\mathrm{L}}$ " quanto maior a quantidade de areia adicionada. Estes mesmos autores questionaram a viabilidade do modelo de linearização de região única para obtenção do " $\mathrm{K}_{\mathrm{L}}$ ", considerando que a adsorção de $\mathrm{P}$ se dá em 3 estádios com energia de ligação distintas, porém concluíram que os valores de " $K_{L}$ " foram mais coerentes relacionados com o modelo de linearização de região única.

O maior FCP máx foi obtido no LVA, visto que a CMAP é constante para o solo e o KL varia em função do conteúdo de $\mathrm{P}$ no solo, o produto das duas variáveis $\left(\mathrm{FCP}_{\text {máx }}\right)$ pode ajudar a elucidar o comportamento do $\mathrm{P}$ no mesmo tipo de solo em função de manejos distintos (HOLFORD; MATTINGLY, 1976).

O P é adsorvido por minerais de argila de carga variável, predominante em regiões tropicais, assim, geralmente, solos com maior conteúdo de argila possuem maior capacidade de reter $\mathrm{P}$ por apresentar maior área de superfície específica e, consequentemente, mais sítios de sorção (MELO; ALEONI, 2009). Dados semelhantes foram observados por Corrêa et al. (2011), avaliando a adsorção de fósforo em Argissolo Amarelo (PA), Latossolo Amarelo (LA), Neossolo Quartzarênico (RQ); Argissolo Vermelho- Amarelo (PVA) e Latossolo Vermelho-Amarelo (LVA); Espodossolo Álico (EP) e Plintossolo Tb Álico (PL), no qual, constataram a amplitude de variação da CMAP em relação ao teor de argila. A heterogeneidade química e de tamanho de partícula entre classes de solos são características que promovem a diferenças de adsorção de P (WANG et al., 2012).

$\mathrm{O} \mathrm{pH}$ influencia diretamente a adsorção de ânions no solo, onde sob condições ácidas, a protonação e solubilização de óxidos pode ocorrer, adiquirindo cargas positivas e, assim, adsorvem os ânions (FERNANDEZ-PAZOS et al., 2013). Deste modo, quanto menor o $\mathrm{pH}$, mais sítios de sorção positivos podem ser gerados, tendendo a adsorver mais 
fosfato, o que não foi encontrado neste trabalho, pois o solo com menor valor de $\mathrm{pH}$ teve os parâmetros de adsorção mais baixo. Provavelmente este achado deve-se a grande diferença textural entre os solos, sendo esta a variável de maior relevância, prevalecendo sobre o pH (ALOVISI et al., 2020).

O solo com maior teor de MO obteve a menor adsorção de fosfato. A correlação negativa entre $\mathrm{MO}$ e os parâmetros de adsorção de $\mathrm{P}$, provavelmente está relacionada com a competição entre os ácidos orgânicos e fosfatos para os sítios de adsorção no colóides (ABDALA et al., 2012). A correlação negativa entre CMAP e Al solúvel pode estar relacionada com a precipitação do $\mathrm{P}$ com o $\mathrm{Al}$, reduzindo a adsorção de $\mathrm{P}$ (LAIR et al., 2009; SOUSA et al., 2012).

\section{CONCLUSÕES}

A isoterma de Langmuir se ajusta bem à adsorção do fósforo e mostra-se de grande eficiência no cálculo dos parâmetros de sorção do P no solo.

A dinâmica de adsorção de $\mathrm{P}$ é influenciada positivamente pelo teor de argila e negativamente pelo teor de $\mathrm{MO} . \mathrm{pH}$ e $\mathrm{Al}^{3+}$ têm papel secundário na dinâmica de adsorção quando comparamos solos de grande diferença textural.

\section{REFERÊNCIAS}

ABDALA, D. B.; GHOSHA, A. K.; SILVA, I. R.; NOVAIS, R. F.; VENEGA, V. H. A. Phosphorus saturation of a tropical soil and related P leaching caused by poultry litter addition. Agriculture, Ecosystems and Environment, v. 162, p. 15-23, 2012. DOI: https://doi.org/10.1016/j.agee.2012.08.004

ALOVISI, A. M. T. Soil factors affecting phosphorus adsorption in soils of the Cerrado, Brazil. Geoderma Regional, v. 22, e00298, 2020. DOI: https://doi.org/10.1016/j.geodrs.2020.e00298

BRADY, N. C.; WEIL, R. R. Elementos da natureza e propriedades do solo. 3 ed. Porto Alegre: Bookman, 2013. 668 p.

CANTARUTTI, R. B.; BARROS, N. F. de; PRIETO, H. E.; NOVAIS, R. F. Avaliação da fertilidade do solo e recomendação de fertilizantes. In: NOVAIS, R. F.; ALVAREZ, V.; BARROS, N. F.; FONTES, R. L. F.; CANTARUTTI, R. B.; NEVES, J. C. L. Fertilidade do solo. Viçosa, MG: Sociedade Brasileira de Ciência do Solo, 2007a. 1017p.

CORRÊEA, R. M.; DO NASCIMENTO, C. W. A.; DA ROCHA, A. T. Adsorção de fósforo em dez solos do Estado de Pernambuco e suas relações com parâmetros físicos e químicos. Acta Scientiarum - Agronomy, Maringá, v. 33, n. 1, p. 153-159, 2011. DOI: http://dx.doi.org/10.4025/actasciagron.v33i1.3129

DE OLIVEIRA, C. M. B.; GATIBONI, L. C.; MIQUELLUTI, D. J.; SMYTH, T. J.; ALMEIDA, J. A. Capacidade máxima de adsorção de fósforo e constante de energia de ligação em latossolo bruno em razão de diferentes ajustes do modelo de langmuir. Revista Brasileira de Ciência do Solo, Viçosa, v. 38, n. 6, p. 1805-1815, 2014.2 DOI: http://dx.doi.org/10.1590/S0100-06832014000600015

EMBRAPA_EMPRESA BRASILEIRA DE PESQUISA AGROPECUÁRIA. Manual de Métodos de Análise de Solo. 3 ed. Brasília: Embrapa, 2017. 573p.

FALCÃO, N. P. S.; SILVA, J. R. A. Phosphorus adsorption characteristics in some central Amazonian soils. Acta Amazonica, Manaus, v. 34, n. 3, p. 337-42, 2004. DOI: http://dx.doi.org/10.1590/S0044-59672004000300001.

FANG, H.; CUI, Z.; HE, G.; HUANG, L.; CHEN, M. Phosphorus adsorption onto clay minerals and iron oxide with consideration of heterogeneous particle morphology. Science of The Total Environment, v. 605-606, p. 357-367, 2017. DOI: https://doi.org/10.1016/j.scitotenv.2017.05.133

FARIAS, D. R.; OLIVEIRA, F. H. T.; SANTOS, D.; ARRUDA, J. A.; HOFFMANN, R. B.; NOVAIS, R. F. Fósforo em solos representativos do estado da Paraíba: Isotermas de adsorção e medidas do fator capacidade de fósforo. Revista Brasileira de Ciência do Solo, Viçosa, v. 33, n. 3, p. 623-632, 2009. DOI: http://dx.doi.org/10.1590/S0100-06832009000300015.

FERNÁNDEZ-PAZOS, M. T.; GARRIDORODRIGUEZ, B.; NÓVOA-MUÑOZ, J. C.; ARIASESTÉVEZ, M.; FERNÁNDEZ-SANJURJO, M. J.; NÚÑEZ-DELGADO, A.; ÁLVAREZ, E. $\operatorname{Cr}(\mathrm{VI})$ adsorption and desorption on soils and biosorbents. Water, Air and Soil Pollution, v. 224, p. 1366, 2012. DOI: 10.1007 / s11270-012-1366-3

GUEDES, R. S.; FERNANDES A. R.; SOUZA E. S.; SILVA, J. R. R. Maximum Phosphorus Adsorption Capacity Adjusted to Isotherm Models in Representative Soils of Eastern Amazon. Communications in Soil Science and Plant Analysis, v. 46, p. 2615-2627, 2015. DOI: https://doi.org/10.1080/00103624.2015.1089264

HOLFORD, I. C. R. Evaluation of soil phosphate buffering indices. Australian Journal of Soil Research, v. 17, n. 3, p. 495-504, 1979 . DOI: https://doi.org/10.1071/SR9790495

LAIR, G. J. F.; ZEHETNER, Z. H.; KHAN, M. H. Phosphorus sorption-desorption in alluvial soils of a young weathering sequence at the Danube River. Geoderma, v. 149, p. 39-44, 2009. DOI: ttps://doi.org/10.1016/j.geoderma.2008.11.011

LIMA, H. N.; MELLO, J. W. V.; SCHAEFER, C. E. G. R.; KER, J. C.; LIMA, A. M. N. Mineralogia e química de três solos ao longo de uma sequência superior da bacia do Alto Solimões, oeste. Revista Brasileira de Ciência do Solo, Viçosa, v. 30, n. 1, p. 59-68, 2006. DOI: http://dx.doi.org/10.1590/S0100-06832006000100007.

MATOS, C. H. D. L.; MELO, V. F.; UCHÔA, S. C. P.; NASCIMENTO, P. P. R. R. N.; PEREIRA, R. A. Phosphorus adsorption in soils under forest and savanna from Northern Amazon, Brazil. Semina: Ciências Agrárias, Londrina, v. 38, n. 5, p. 2909-2919, set./out. 2017. DOI: DOI: 10.5433/1679-0359.2017v38n5p2909

MELO, V. F.; SCHAEFER, C. E. G. R.; FONTES, L. E. F.; CHAGAS, A. C.; LEMOS JÚNIOR, J. B.; ANDRADE, R. P. Solos da área indígena Yanomami no médio Rio Catrimani, Roraima. Revista Brasileira de Ciência do Solo, Viçosa, v. 34, n. 2, p. 487-496, 2010. DOI: http://dx.doi.org/10.1590/S0100-06832010000200022.

MELO, V. de F.; ALLEONI, L. R. F. Química e mineralogia do solo. Parte II: Aplicações. Viçosa: Sociedade Brasileira de Ciência do Solo, 2009. 1381p.

OLIVEIRA, M. B.; GATIBONI, L. C.; MIGUELLUTI, D. J.; SMYTH, T. J.; ALMEIDA, J. A. Capacidade máxima de adsorção de fósforo e constante de energia de ligação em Latossolo Bruno em razão de diferentes ajustes do modelo de Langmuir. Revista Brasileira de Ciência do 
Solo, Viçosa, v. 38, n. 6, p. 1805-1815, 2014. DOI: https://doi.org/10.1590/S0100-06832014000600015.

PARFITT, R. L. Phosphate reactions with natural allophane, ferrihydrite and goethite. Journal of Soil Science, v. 40, p. 359-369, 1989. DOI: https://doi.org/10.1111/j.13652389.1989.tb01280.x

PARFITT, R. L. Anion adsorption by soils and soil materials. Advances Agronomy, v. 30, p. 1-50, 1979. DOI: https://doi.org/10.1016/S0065-2113(08)60702-6

PELUCO, R. R.; JÚNIOR, J. M.; SIQUEIRA, D. S.; PEREIRA, G. T.; BARBOSA, R. S.; TEIXEIRA, D. B. Mapeamento do fósforo adsorvido por meio da cor e da suscetibilidade magnética do solo. Pesquisa Agropecuária Brasileira, Brasília, v. 50, n. 3, p. 259-266, 2015. DOI: https://doi.org/10.1590/S0100204X2015000300010

RADAMBRASIL. FOLHA SF.21 CAMPO GRANDE. Ministério das Minas e Energia - Departamento Nacional da Produção Mineral. Levantamentos de Recursos Naturais. Rio de Janeiro: RADAMBRASIL, v. 28, 1982.

RHEINHEIMER, D. S.; ANGHINONI, I.; CONTE, E. Sorção de fósforo em função do teor inicial e de sistemas de manejo de solos. Revista Brasileira de Ciência do Solo, Viçosa, v. 27, p. n. 1, 41-49, 2003. DOI: https://doi.org/10.1590/S0100-06832003000100005

ROSS, S. M. Organic matter in tropical soils: current conditions, concerns, and prospects for conservation. Progress in Physical Geography, v. 17, n. 3, p. 365-305, 1993.

DOI: https://doi.org/10.1177\%2F030913339301700301

SCHIAVO, J. A.; PEREIRA, M. G.; MIRANDA, L. P. M.; NETO, A. H. D.; FONTANA, A. F. Caracterização e classificação de solos desenvolvidos de arenitos da formação Aquidauana-MS. Revista Brasileira de Ciencia do Solo, Viçosa, v. 34, n. 3, p. 881-889, 2010.

SOUSA, A. F.; BRAGA, T. P.; GOMES, E. C. C.; VALENTINI, A.; LONGHINOTTI, E. Adsorption of phosphate using mesoporous spheres containing iron and aluminum oxide. Chemical Engineering Journal, v. 210, p. 143-149, 2012. DOI: https://doi.org/10.1016/j.cej.2012.08.080

SOUSA, D. M. G.; LOBATO, E. Cerrado Correção e adubação. 2 ed. Brasília-DF: Embrapa Informação Tecnológica, 2004. 416p.

WANG, Y.; SHEN, Z.; NIU, J.; LIU, R. Adsorption of phosphorus on sediments from the Three-Gorges Reservoir (China) and the relation with sediment compositions. Journal of Hazardous Materials, v. 162, n. 1, p. 92-98, 2009. DOI: 10.1016/j.jhazmat.2008.05.013

WANG, X.; ZHANG, L.; ZHANG, H.; WU, X.; MEI, D. Phosphorus adsorption characteristics at the sedimentwater interface and relationship with sediment properties in Fushi reservoir, China. Environmental Earth Science, v. 67, p. 15-22, 2012. DOI: $10.1007 /$ s12665011-1476-z 